\title{
COMPARAÇÃO DO EFEITO DE PIGMENTOS DE ORIGEM FÚNGICA E CORANTES COMUMENTE USADOS COMO MEDIADORES ELETROQUÍMICOS SOBRE O CRESCIMENTO MICROBIANO E A ATIVIDADE OXIDASICA DE Rhizopus microsporus var. chinensis.
}

\author{
P. H. da SILVA ${ }^{1,2} ;$ K. V. MORANT ${ }^{1,2} ;$ G. M. C. TAKAKI ${ }^{2}$; C. E. la ROTTA H. ${ }^{2}$ \\ ${ }^{1}$ Universidade Católica de Pernambuco, Departamento de Engenharia Química \\ ${ }^{2}$ Universidade Católica de Pernambuco, Núcleo de Pesquisas em Ciências Ambientais e Biotecnologia \\ E-mail para contato: phenriquedasilva@yahoo.com
}

\begin{abstract}
RESUMO - As oxidases usam o oxigênio molecular como aceptor de elétrons em reações de oxidação. $\mathrm{O}$ transporte de elétrons entre os biocatalisadores, durante a redução catódica do oxigênio, e os eletrodos pode ser melhorado através de mediadores eletroquímicos. Contudo, seu uso é limitado devido a elevada toxicidade e custo. Como uma alternativa para mediadores sintéticos, algumas biomoléculas vem sendo estudadas. Este trabalho, comparou o uso de pigmentos fúngicos com outros mediadores eletroquímicos durante o crescimento de Rhizopus microsporus var. chinensis e que está sendo usado na redução in-situ de oxigênio em biocátodos. Em geral foi observado que estes pigmentos fúngicos não causaram nenhuma inibição no crescimento do fungo. Por outro lado, os corantes trifenilmetano avaliados causaram inibição. Os resultados obtidos sugerem que seria possível o uso concomitante de mediadores eletroquímicos de origem fúngica durante o cultivo in-situ de fungos com atividade oxidásica em BCC ou em sistemas simbióticos.
\end{abstract}

\section{INTRODUÇÃO}

O uso de biocélulas a combustível tem sido limitado, devido aos altos custos de produção e purificação e ao curto tempo de vida útil, devido à inativação dos biocatalizadores. Carbajosa et al. (2010). Para permitir a transferência de elétrons para o eletrodo, são usados compostos solúveis que agem como mediadores eletroquímicos que possuem um potencial redox apropriado e que podem alcançar a superfície dos eletrodos.

Recentemente, bioeletrodos enzimáticos têm sido o centro de muitas pesquisas devido a suas vantagens em relação a eletrodos abióticos. Contudo, a maioria dos biocatalizadores que estão em uso tem como característica própria não possibilitar uma transferência eletrônica fácil, causando a perda da carga na forma de elétrons coletados a partir do substrato. Portanto, sistemas eletroquímicos diretos ou mediados, tem sido desenvolvidos para alcançar essa transferência eletrônica com maior eficiência. La Rotta e Gonzáles. (2013), Brunel et al. 
(2007). Por outro lado, uma vasta quantidade de moléculas que incluem: compostos organometálicos (ex. ferroceno), derivados de quinonas (ex. hidroquinonas), corantes tiazínicos (ex. azul de metileno), compostos azo (ex. ABTS) e mais recentemente corantes trifenilmetano (ex. verde de bromocresol). La Rotta et al. (2011). Tem sido usados extensivamente na literatura como transportadores de elétrons. Apesar disso, essas moléculas tem algumas limitações, especialmente devido ao seu alto custo e toxicidade elevada. Como uma alternativa para os mediadores sintéticos, algumas biomoléculas também vem sendo exploradas, incluindo pigmentos bacterianos e fúngicos. Entre esses pigmentos microbianos podem se encontrar azafilonas, antraquinonas, oxipolienos, carotenos, clorofilas, melaninas e xantofilas. Todos estes metabolitos microbianos são conhecidos por participar em diversos processos bioquímicos incluindo: proteção contra os efeitos dos danos potenciais da luz solar, em particular alguns comprimentos de onda UV; manter a integridade dos esporos de dispersão e dos esporos inativos, proporcionando proteção contra a radiação e dessecação; reserva de energia durante a fase de esporos ou de esporângios de formação; inibidores enzimáticos que podem interferir no processo de crescimento do fungo; reguladores dos processos redox agindo como antioxidantes e durante os processos oxidativos. Forgaty e Tobin (1996).

O objetivo deste trabalho foi avaliar o crescimento do micro-organismo Rhyzopus microsporus var. chinensis produtor de enzimas oxidásicas em conjunto com pigmentos fúngicos para um possível cultivo concomitante in-situ em biocátodos de biocélulas a combustível de micro-organismo produtores de pigmentos e de enzimas oxidásicas sem interferir no crescimento e rendimento de ambos.

\section{METODOLOGIA}

\subsection{Cepas fúngicas}

Inicialmente doze cepas fúngicas isoladas da Caatinga, foram obtidas através do banco de culturas da Universidade Católica de Pernambuco pela RENNORFUN: Rhyzopus microsporus var. chinensis SIS-31, Rhyzopus microsporus var. microsporus SIS-39, Aspergillus sp. SIS-18, Penicillium sp. SIS-27, Aspergillus sp. SIS-4(E), Aspergillus sp. SIS7, Eupenicillium orangecolony, Eupenicillium sp A2P1 10-3, Talaromyces sp A2P1 10-4 G, Talaromyces sp. A2P1 10-3, Penicillium sp. SIS-21, Penicillium sp. CP1 10-3 H. A seleção das cepas para o experimento foi baseada na avaliação visual de excreção de pigmentos no meio de cultura, sendo cultivadas em ágar Sabouraud por 72 horas à $28^{\circ} \mathrm{C}$.

\subsection{Seleção dos meios e separação dos pigmentos}

A seleção preliminar dos pigmentos foi realizada utilizando quatro meios sólidos diferentes: Meio Olga (conforme o trabalho de Olga et al. (1998)) ( $\left.\mathrm{g} \mathrm{L}^{-1}\right)$ - Glicose 10,0; Peptona 3,0; $\mathrm{KH}_{2} \mathrm{PO}_{4}$ 0,6; $\mathrm{ZnSO}_{4} 0,001 ; \mathrm{K}_{2} \mathrm{HPO}_{4}$ 0,4; $\mathrm{FeSO}_{4} 0,0005 ; \mathrm{MnSO}_{4} 0,05 ; \mathrm{MgSO}_{4}$ 0,5; e Ágar 20,0. Meio Olga Modificado $\left(\mathrm{g} \mathrm{L}^{-1}\right)$ - Glicerol 20,0; Peptona 3,0; $\mathrm{KH}_{2} \mathrm{PO}_{4}$ 0,6; $\mathrm{ZnSO}_{4}$ 0,001; $\mathrm{K}_{2} \mathrm{HPO}_{4}$ 0,4; $\mathrm{FeSO}_{4} 0,0005, \mathrm{MnSO}_{4}$ 0,05; $\mathrm{MgSO}_{4}$ 0,5; e Ágar 20,0. Meio Palanivel (conforme o trabalho de Palanivel et al. (2010)) (g L $\left.{ }^{-1}\right)$ - Glicerol 20,0; $\mathrm{NH}_{3} \mathrm{SO}_{4}$ 1,$0 ; \mathrm{KH}_{2} \mathrm{PO}_{4} 0,8 ; \mathrm{ZnSO}_{4} \mathrm{H}_{2} \mathrm{O} 0,8 ; \mathrm{K}_{2} \mathrm{HPO}_{4} 1,4 ; \mathrm{FeCl}_{3} 6 \mathrm{H}_{2} \mathrm{O} 0,8 ; \mathrm{NaMoO}_{4} 2 \mathrm{H}_{2} \mathrm{O} 0,8 ; \mathrm{MgSO}_{4}$ $7 \mathrm{H}_{2} \mathrm{O}$ 0,5; $\mathrm{MnSO}_{4} 2 \mathrm{H}_{2} \mathrm{O}$ 0,4; $\mathrm{CuSO}_{4} 0,08$ e Ágar 20,0. Meio Nutriente Modificado $\left(\mathrm{g} \mathrm{L}^{-1}\right)$ - 
Glicerol 20,0; Peptona 6,0; Extrato de Carne 4,0; Ágar 20,0. A seleção do meio de cultura foi baseada na produção de pigmento e intensidade da cor difundida no meio. Todas as cepas foram cultivadas por um período de 72 horas à $28^{\circ} \mathrm{C}$. Após a seleção, apenas as cepas mais produtoras de pigmentos foram usadas na etapa seguinte de cultivo utilizando mais adequado ou selecionado, durante 240 horas, $180 \mathrm{rpm}$ à $28^{\circ} \mathrm{C}$. Os pigmentos obtidos após a fermentação foram separados pelo caldo metabólico, por centrifugação por 10 minutos a $4500 \mathrm{rpm}$.

\subsection{Análise espectrofotométrica}

Frascos de penicilina contendo $20 \mathrm{~mL}$ de cada caldo metabólico livre de células foram liofilizados até a secagem total restando, apenas uma pasta contendo pigmento em cada frasco. A partir destas pastas forma pesados $10 \mathrm{mg}$ de cada pigmento e dissolvidos em $1 \mathrm{~mL}$ de agua milliq para obtenção de soluções com as concentrações conhecida de $10 \mathrm{mg} \mathrm{mL} \mathrm{m}^{-1}$. Inicialmente, foram utilizadas soluções concentradas de cada pigmento, para determinar o comprimento de onda máximo, Tabela 1, através da varredura no espectro UV-Vis de 220 a $880 \mathrm{~nm}$ em cubetas de quartzo de $1 \mathrm{~cm}$ de comprimento. A seguir foram realizadas diluições de diferentes concentrações e utilizadas na elaboração de curvas de calibração que permitiram estimar a quantidade de pigmento presente em cada cultura fúngica no caldo metabólico bruto.

Tabela 1.Absorbâncias dos pigmentos observadas nos comprimentos de onda de $\mathrm{ABS}_{\max }$

\begin{tabular}{|c|c|c|c|}
\hline Vermelho & Verde & Laranja & Amarelo \\
\hline$\lambda_{\max } 492 \mathrm{~nm}$ & $\lambda_{\max } 400 \mathrm{~nm}$ & $\lambda_{\max } 370 \mathrm{~nm}$ & $\lambda_{\max } 380 \mathrm{~nm}$ \\
\hline
\end{tabular}

\subsection{Fermentação e espectrofotometria}

Os pigmentos solubilizados no caldo metabólico foram novamente submetidos a espectrofotometrias de varredura para identificação dos picos de máxima absorção. Inicialmente foram feitos estoques de $100 \mathrm{ppm}$ para todos os pigmentos para solubilização em $200 \mathrm{ml}$ de meio de cultura Olga, otimizado para o cultivo do micro-organismo Rhyzopus microsporus var. chinensis, para uma concentração de $10 \mathrm{ppm}$. Com o meio de cultura inoculado com o pigmento e o micro-organismo, realizou-se a fermentação por 240 horas a uma rotação de $180 \mathrm{rpm}$ a $28^{\circ} \mathrm{C}$, sendo retiradas amostras diárias para realização de análises espectrofotométricas para consumo de pigmento.

\subsection{Ciclovoltametria}

O objetivo dessa técnica é identificar os picos de redução e de oxidação do analíto neste caso o pigmento solubilizado numa solução eletrolítica de suporte adequada, podendo ser identificados os picos redox ou pares de redução e oxidação (pares redox) mostrando reversibilidade destes pares num potencial específico aplicado. Para esta técnica, os pigmentos foram liofilizados. Foram utilizadas células eletroquímicas contendo como eletrólitos de suporte, cloreto de potássio $(\mathrm{KCl})$ e ácido fosfórico $\left(\mathrm{H}_{3} \mathrm{PO}_{4}\right)$, ambas de $10 \mathrm{mmol}$ $\mathrm{L}^{-1}$. O sistema eletroquímico de análise esteve formado por um eletrodo de trabalho de carbono vítreo de $0,5 \mathrm{~cm}$ de diâmetro, um contra eletrodo de fio platina de $1,0 \mathrm{~cm}$ e como eletrodo de referência $\mathrm{Ag} \mid \mathrm{AgCl}_{2} \mathrm{em} \mathrm{KCl}$ saturado. Varreduras de potencial foram realizadas de $-1,0$ a $1,0 \mathrm{~V}$ com velocidades de $0,1 \mathrm{~V} \mathrm{~s}^{-1}$. Uma célula eletroquímica de $15 \mathrm{ml}$ foi utilizada 
em conjunto com um potenciostato PalmSens ${ }^{\circledR}$ LabSolutions com software de aquisição de dados PS-Trace® 4.2. Fernández-Sánchez et al. (2002); Rachinski et al. (2010)

\subsection{Inibição do crescimento, consumo de pigmentos e atividade enzimática}

Para a avaliar inibição do crescimento em placa, foi inoculada uma suspensão espórica de 2,5 $\times 10^{5}$ esporos por ml do micro-organismo no meio morno ainda líquido e distribuída em placa de petri para crescimento uniforme. Foi adicionado no centro da placa, com o meio frio e solidificado, discos de papel xarope de $1,0 \mathrm{~cm}$ de diâmetro, embebidos com $100 \mu \mathrm{l}$ de uma solução em água de $10 \mathrm{ppm}$ de cada pigmento. O micro-organismo foi incubado durante um período de 72 horas a uma temperatura de $28^{\circ} \mathrm{C}$, e foram feitas medições diárias de halo de inibição. Para a avaliação de crescimento em meio líquido, o micro-organismo foi cultivado inicialmente em Meio Olga sólido, por um período de $72 \mathrm{~h}$ a $28^{\circ} \mathrm{C}$. Após esse período de crescimento uniforme do micro-organismo na placa, foram retirados discos de $0,8 \mathrm{~mm}$ de diâmetro da placa usados para inocular $200 \mathrm{ml}$ de meio Olga líquido contendo $10 \%$ de pigmento solubilizado, sendo a sua concentração final no meio de $10 \mathrm{ppm}$. O mesmo foi realizado para a avaliação de produção enzimática em meio Olga com pigmentos. Foram realizadas fermentações para todos os pigmentos por um período de $240 \mathrm{~h}$ a $28^{\circ} \mathrm{C}$ e com uma agitação de $180 \mathrm{rpm}$, sendo retiradas amostras diárias para análise de consumo de pigmento e de atividade enzimática por espectrofotometria. O método para determinação da atividade enzimática é realizado por meio da oxidação do pirogalol (solução de $10 \mathrm{mmol} \mathrm{L}^{-1}$ ) para a purpurogalina, a atividade oxidásica foi determinada pela formação de um composto de cor amarela que é lido no espectrofotômetro a $420 \mathrm{~nm}$, quanto mais intensa for sua cor, mais pirogalina foi formada, indicando assim que a enzima é mais ativa. TRIANA et al. (2005).

\section{RESULTADOS E DISCUSSÃO}

\subsection{Seleção dos micro-organismos e meios de cultura}

Tabela 2. Pigmentos excretados pelos correspondentes fungos filamentosos, após no meio de cultura otimizado.

Tabela 2 - Micro-organismos selecionados como produtores de pigmentos

\begin{tabular}{cc}
\hline Micro-organismo & Pigmento \\
\hline Aspergillus sp. SIS-4(E) & Vermelho \\
Penicillium CP1 $10-3 \mathrm{H}$ & Verde \\
Talaromyces A2P1 10-4 G & Laranja \\
Penicillium sp. SIS 21 & Amarelo \\
\hline
\end{tabular}

Os Pigmentos foram melhor produzidos e excretados no meio nutriente modificado. Esse resultado mostrou-se interessante desde o ponto de vista biotecnológico, pois visa também à reutilização e aproveitamento de glicerol para a geração de produtos de valor agregado, que neste caso corresponderia à produção de pigmentos com propriedades 
eletrogênicas. Na Figura 1, é possível notar a produção e excreção dos pigmentos no meio e cultura.

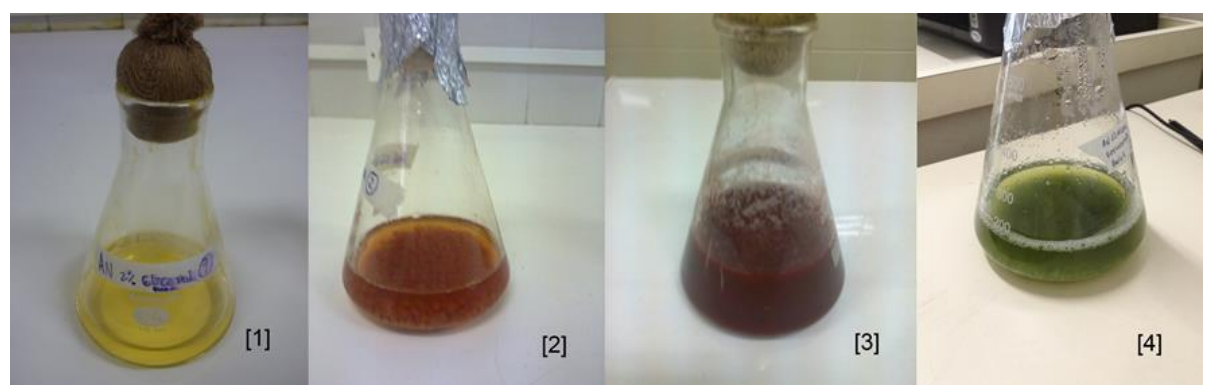

Figura 1 - Exemplo das culturas fúngicas submersas em Meio Nutriente Modificado que apresentaram produção significativa de pigmentos. ([1] Penicillium sp. SIS 21.[2] Talaromyces A2P1 10-4 G.[3] Aspergillus sp. SIS-4(E). [4] Penicillium CP1 10-3 H.)

\subsection{Voltametria cíclica}

As análises preliminares por voltametria cíclica mostraram em geral um comportamento semelhante com quase todos os pigmentos, sendo na sua maioria observados dois picos de oxidação e três picos de redução como na Figura 2. Indicando a presença de um pico de redução irreversível. Embora estes estudos eletroquímicos sejam preliminares, em geral se observa que todos os pigmentos possuem uma boa capacidade para transferência e transporte de carga, mesmo sendo observada a irreversibilidade de um dos picos, como indicado em DA SILVA et al. (2014).

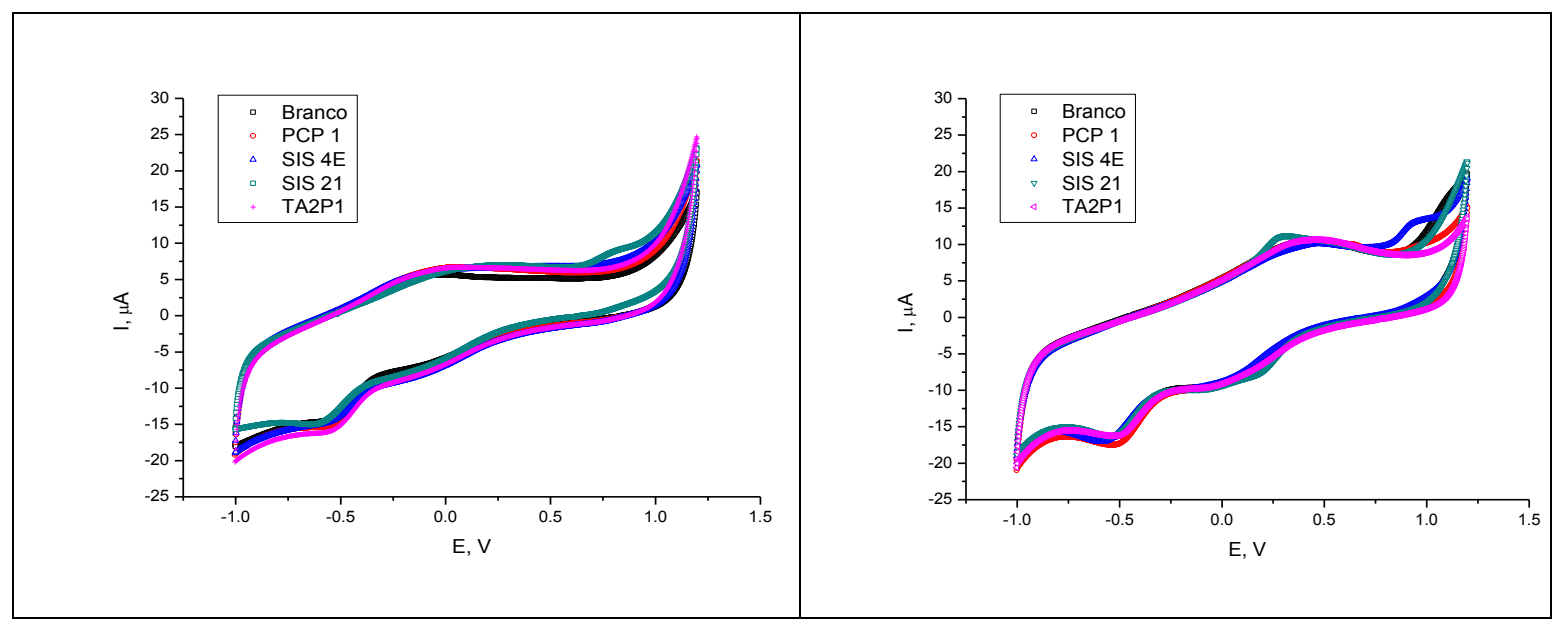

Figura 2 - Curvas de ciclovoltametria obtidas para os pigmentos fúngicos em eletrólitos de suporte de $\mathrm{KCl}$ (à esquerda) e $\mathrm{H}_{3} \mathrm{PO}_{4}$ (à direita) $10 \mathrm{mmol} \mathrm{L}^{-1}$.

\subsection{Inibição do crescimento}

Os resultados ao final do período de incubação de 72 horas mostraram que pigmentos fúngicos desde o primeiro dia, não apresentaram nenhum tipo de inibição sobre o crescimento 
do Rhyzopus microsporus var. chinensis Por outro lado, os pigmentos sintéticos, azul de bromotimol e azul de metileno, apresentaram halo de inibição de $0,5 \mathrm{~cm}$ nas primeiras 48 horas, que foram diminuindo até que no final das 72 horas, o halo de inibição presente foi um pouco maior que $0,1 \mathrm{~cm}$. Na Figura 3 foi possível notar a grande diferença, no período de 48 horas, entre a inibição causada pelo corante sintético e biológico que não causou inibição, como exemplo o corante azul de bromotimol e o pigmento fúngico verde respectivamente.

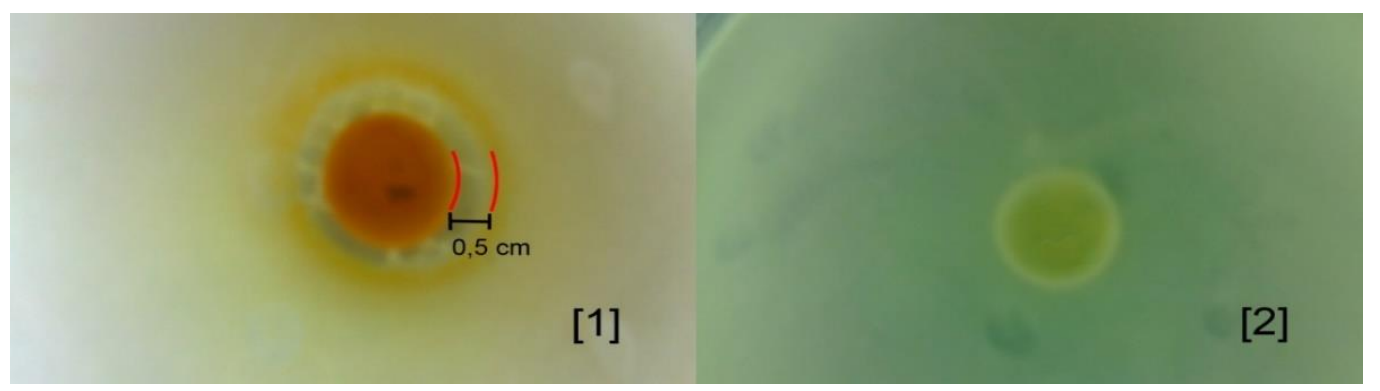

Figura 3 - Micro-organismo Rhyzopus microsporus var. chinensis [1] halo de inibição com o pigmento inorgânico azul de bromotimol no tempo de 48 horas. [2] Inexistência de halo de inibição do pigmento verde no tempo de 48 horas.

\subsection{Consumo de pigmentos}

As amostras retiradas nas fermentações, foram utilizadas para leitura no espectrofotômetro e através de varredura espectrofotométrica foi possível identificar as curvas de absorção das fermentações. As leituras mostraram um decaimento médio de 0,2 nm na absorbância dos pigmentos inorgânicos (azul de bromotimol e azul de metileno) durante o período de $240 \mathrm{~h}$, enquanto que nos pigmentos fúngicos, foi observada uma constância nas leituras, com uma variação média de menos de 0,002 nm durante todo o período da fermentação. Mostrando que existe uma inibição com relação aos pigmentos inorgânicos sendo os pigmentos fúngicos melhores para serem usados no crescimento com outro microorganismo

\subsection{Efeito sobre a atividade enzimática}

Um efeito inicial, é a incapacidade de produção de enzima do Rhyzopus microsporus var. chinensis em meio com glicerol, sendo apenas expressada produção em meio com glicose sendo maior em meio Olga MORANT et al. (2014). As leituras mostraram um comportamento estável da maioria dos pigmentos. Porém, nos experimentos contendo os pigmentos laranja, amarelo e no azul de bromotimol, foi identificado na um aumento na atividade enzimática em $144 \mathrm{~h} 192 \mathrm{~h}$ e $216 \mathrm{~h}$ respectivamente, como é mostrado na Figura 4. O pigmento laranja teve um pico de atividade de quase $70 \mathrm{kUI}$, o pigmento amarelo um pico de um pouco mais de $60 \mathrm{kUI}$, já no azul de bromotimol foi observado um pico máximo de um pouco mais de $120 \mathrm{kUI}$. Mesmo sendo o azul de bromotimol o pigmento que permitiu a enzima uma maior atividade, foi mostrando no experimento de crescimento, que o pigmento em questão inibiu o crescimento do microrganismo. 


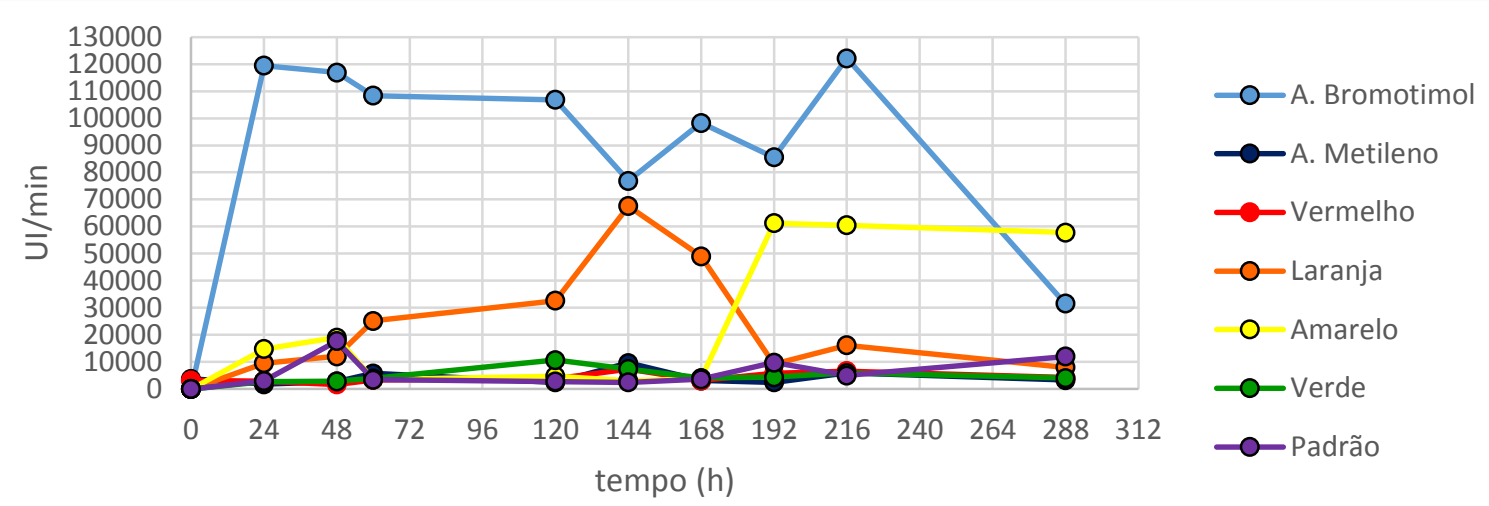

Figura 4 - Gráfico da atividade enzimática com os respectivos pigmentos em UI/min pelo Tempo em horas

\section{CONCLUSÕES}

Todas as cepas escolhidas apresentaram uma elevada excreção de pigmento no meio de cultura. No entanto, foi observado que os micélios continham também uma elevada concentração de pigmento não excretado. Por tanto, seria possível melhorar ainda mais a produção deste tipo de compostos investindo nos métodos de extração ou na produtividade da excreção dos pigmentos ao meio de cultura. $\mathrm{O}$ trabalho mostrou-se muito promissor, pois a maioria dos compostos utilizados como transportadores de eletros em sistemas eletroquímicos possuem alta toxicidade e reações indesejáveis com outras substancias presentes nos meios de cultura o que diminui ainda mais sua eficiência na transferência de elétrons DA SILVA et al. (2014). Também foi observado, o melhor uso dos pigmentos fúngicos em relação ao crescimento e a atividade enzimática. $\mathrm{O}$ experimento contendo maior atividade enzimática, foi o que continha o pigmento azul de bromotimol. Mas como sua inibição sobre o crescimento do micro-organismo foi alta, não seria bem aproveitado para uso de crescimento conjunto com o Rhyzopus microsporus var. chinensis, sendo os pigmentos fúngicos os de melhor aplicabilidade para um crescimento in-situ.

\section{AGRADECIMENTOS}

Os autores agradecem o apoio financeiro dado pelos programas de Desenvolvimento Científico Regional através do projeto DCR No.0008-1.06/11 e do Programa de Bolsas de Iniciação Científica Conselho Nacional de Pesquisa - CNPq e da Fundação para o Amparo à Pesquisa e Tecnologia do Estado de Pernambuco - FACEPE

\section{NOMENCLATURA}

$\lambda_{\text {max: }}$ comprimento de onda máximo; ${ }^{\circ} \mathbf{C}$ : graus célsius; $\mathbf{g}$ : grama; g.L $\mathbf{L}^{\mathbf{- 1}}$ : gramas por litro; in-situ: Se refere ao mesmo recipiente; $\mathbf{m g}$ : miligrama; $\mathbf{m L}$ : mililitro; $\mathbf{m m o l} . \mathbf{L}^{\mathbf{- 1}}$ : milimol por litro; nm: nanômetros; ppm: partes por milhão; rpm: rotações por minuto; UI: unidade enzimática internacional; UV-Vis: Ultravioleta visível; V: volts; $\mathbf{V} \cdot \mathbf{s}^{\mathbf{- 1}}$ : volts por segundo.

\section{REFERÊNCIAS}


BRUNEL L, DENELE J, SERVAT K, /KOKOH K B, JOLIVALT C, INNOCENT C, CRETIN M, ROLLAND M, TINGRY S. Oxygen transport through laccase. Electrochemistry Communications. v. 9, No 2, p. 331-336, 2007.

CARBAJOSA S, MALKI M, CAILLARD R, LOPEZ MF, PALOMARES FJ, MARTINGAGO JA, et al. Electrochemical growth of Acidithiobacillus ferrooxidans on a graphite electrode for obtaining a biocathode for direct electrocatalytic reduction of oxygen. Biosens Bioelectron. v. 26, p. 877-80. 2010

DA SILVA, P. H.; MORANT, K. V.; CAMPOS-TAKAKI, G. M.; LA ROTTA, C. E. Production of electrogenic pigments from new fungal sources applied as electron shuttles in biofuel cells. BioMicroWorld 2013. 2014.

FERNÁNDEZ-SÁNCHEZ, C.; TZANOV, T.; GÜBITZ, G. M.; CAVACO-PAULO, A. Voltammetric monitoring of laccase-catalysed mediated reactions. Bioelectrochemistry $\mathrm{v}$. 58, p. 149-156, 2002.

FOGARTY V, TOBIN M. Fungal melanin and their interactions with metals. Enz. Microb. Technol. v. 19, No 4, p. 311-317, 1996.

LA ROTTA CE, CINICIATO G, GONZÁLEZ ER. Triphenylmethane dyes, an alternative for mediated eletronic transfer systems in glucose oxidase biofuel cells. Enz. Microb. Technol.v 48, p. 487-497, 2011.

LA ROTTA CE., GONZÁLEZ ER. Synthesis and Characterization of Chemical Modified Carbon-Chitosan Composites Applied to Glucose Oxidase Fuel Cells J. Electrochem. Soc. 160(1): G37-G45, 2013.

MORANT, K. V., DA SILVA, P. H.; CAMPOS-TAKAKI, G. M.; LA ROTTA, C. E. Screening for new fungi with oxidasic activity applied in-situ as biocatalysts for biofuel cells. BioMicroWorld 2013. 2014

OLGA VKS, ELENA VS, VALERIA PG, OLGA VM, NATALIA VL, AIDA ND, ALEXANDER IJ, ALEXANDER M, Purification and characterization of the constitutive form of laccase from basidiomycete Coriolus hirsutus and effect of inducers on laccase synthesis. Biotechnol. Appl. Biochem. v. 28, p. 47-54, 1998.

PALANIVEL V, KAMALA-KANNAN S, BALACHANDAR V, LAKSHMANAPERUMALSAMY P, CHAE J, OH B. Natural pigment extraction from five filamentous fungi for industrial applications and dyeing of leather. Carbohydrate Poly. v. 79, p. 262-268, 2010.

RACHINSKI, S.; CARUBELli, A.; MANGONI, A.P.; MANGRICH, A.S. Pilhas de Combustíveis Microbianas Utilizadas na Produção de Eletricidade a Partir de Rejeitos Orgânicos: Uma Perspectiva de Futuro Quim. Nova. v. 33, No. 8, p. 1773-1778, 2010.

TRIANA, G.; BÁRBARA E.; PEÑA PINO, ROBERTO DE LA; RODRÍGUEZ SOSA, VÍCTOR; SALDAÑA BERNABEU, ALBERTO; GARCÍA PIÑEIRO, JOSÉ C. Efecto del Vimang Sobre la Actividad Serica de Enzimas Antioxidantes en la Periodontitis Experimental. Instituto Superior de Ciencias Médicas de la Habana. Revista Habanera de Ciencias Médicas, v. 4, No. 3, 2005. 